\title{
Strain-Differentiated Circadian and Ultradian Rhythms in Locomotor Activity of the Laboratory Rat
}

\author{
D. Büttner ${ }^{1}$ and F. Wollnik ${ }^{2}$ \\ Received 11 Feb. 1983-Final 6 Oct. 1983
}

Spontaneous locomotor activity $(L A)$ was recorded in five inbred strains of laboratory rats (ACI/Ztm, AS/Ztm, BH/Ztm, BS/Ztm, LEW/Ztm) under light-dark (LD 12:12), continuous-dark (DD), and continuous-light (LL) conditions. Strain-dependent differences in $L A$ were observed under each light condition. Under LD conditions, 24-h rhythms were found in the LA pattern of each strain studied. In addition, the BH strain showed a clear bimodal, and the LEW strain a trimodal, activity pattern. Spectral anal$y$ sis of the activity patterns revealed distinct 4-and 4.8-h ultradian rhythms in the LEW strain and 12-, 6-, 4.8-, and 4-h rhythms in the BH strain. These strain-characteristic patterns persisted under continuous-dark conditions, therefore demonstrating their endogenous character. Under continuous-bright-light conditions the circadian rhythms decreased to a nonsignificant level in the AS and BS strains. This was not the case, however, in all animals of the $L E W$ and $B H$ strains. Despite the rapid decrease in the 24-h rhythm under LL, the LEW and BH strains showed significant ultradian rhythms.

KEY WORDS: circadian and ultradian rhythms; strain differences; locomotor activity; laboratory rat.

\section{INTRODUCTION}

There is no doubt that daily rhythms in behavioral and physiological activity have evolved as an adaptive response to the 24-h fluctuations in

This work was supported by a grant from Deutsche Forschungsgemeinschaft (SFB 146).

${ }^{1}$ Zentrales Tierlabor für Experimentelle Medizin im Klinikum der Gesamthochschule Essen, Hufelandstr. 55, D-4300 Essen, FRG.

${ }^{2}$ Institut für Versuchstierkunde im Zentralen Tierlaboratorium der Medizinischen Hochschule Hannover, D-3000 Hannover, FRG. 
the environment and are now genetically fixed in many organisms (Daan, 1981). Comparative studies have, additionally, revealed that substantial differences in circadian systems exist even among closely related species.

Although numerous domesticated species demonstrate a high degree of genetic variability within their own species regarding physiological factors, differences in rhythms between individuals or between strains have been shown in only a few species including Drosophila, Neurospora, and $M u s$.

Genes which control some aspects of circadian rhythms have been identified by mutational analysis in Drosophila (Konopka and Benzer, 1971) and Neurospora (Feldmann and Hoyle, 1973). In mammalian species inbred-strain comparisons have been done to analyze food and water consumption (Possidente and Hegmann, 1980), enzyme activity, and rhythms in mice (Peleg et al., 1982) and the relation of the daily patterns of locomotor activity (LA) and catecholamines in rats (Lemmer et al., 1981). Recent experimental evidence suggests strain-dependent differences in the LA patterns of laboratory rats.

Although most investigations on LA patterns are done from the aspect of circadian rhythms, observations involving bimodal and trimodal distributions in LA, heart rate, and body temperature of the laboratory rat have been completed (Büttner and Wollnik, 1982; Büttner and Plonait, 1980; Homna and Hiroschige, 1978a,b,c; Stephan, 1983).

Rhythmic differences within one species cannot be explained solely by inconsistent data sampling and processing or by exogenous influences during the testing period. By observing animals of different origins, the genetic background is also taken into account. In order to prove the hypothesis of genetic-based differences in LA rhythms, LA was measured in five different inbred strains of laboratory rats under identical conditions.

\section{MATERIALS AND METHODS}

\section{Genetic Data}

Male rats of the following inbred strains were used: ACI/Ztm, AS/ $\mathrm{Ztm}, \mathrm{BH} / \mathrm{Ztm}, \mathrm{BS} / \mathrm{Ztm}$, and $\mathrm{LEW} / \mathrm{Ztm}$. The animals were bred in brother-sister mating in our laboratory for about 8 years and were tested for histocompatibility loci by skin transplantation and electrophoresis of organ and serum esterases. The animals were free of those pathogens specified in the GV-Solas list (GV-Solas, 1977).

\section{Maintenance Conditions}

The animals were kept in a separated animal room under controlled environmental conditions (room temperature, $22 \pm 1^{\circ} \mathrm{C}$; relative humid- 
ity, $55 \pm 5 \%$ ). During the LA measurements the animals were kept individually in polycarbonate cages (Makrolon type III, $55 \times 33 \times 20$ $\mathrm{cm})$ on sterile wooden granules. A pelleted diet, Altromin $1324(19 \%$ crude protein, $4 \%$ crude fat, $6 \%$ crude fiber, $7 \%$ ash, and $50.5 \%$ nitrogen-free extract), and tap water from Makrolon bottles was available ad libitum. Before each measuring period the animals were provided with new cages and enough food and water to subsist on for 2 weeks. This way, LA could be measured continuously for 10 days without disturbing the animals.

\section{Measurement of LA and Data Processing}

LA was measured with an electronic movement analyzer which, operating on a capacitant system (Plonait et al., 1982), supplies a continuous signal proportional to the horizontal movement of the animal. The output was recorded at 10 -s intervals by a microcomputer. Five-minute mean values were calculated from these 10 -s intervals. Further calculations were then based on the 5-min averages.

Due to the varying body weights of the animals, the output signals from the capacitant measuring system varied in intensity. The measurements of LA were standardized by setting the overall mean value of each animal at $100 \%$.

In order to examine the periodicity of the rhythmical fluctuations of LA, we used the "generalized harmonic spectral analysis" described by Halberg and Panofsky (1961) and Panofsky and Halberg (1961). This method has the advantage of reducing some of the statistical uncertainties associated with classical methods of harmonic analysis. It evaluates the statistical significance of a given rhythm and allows pooling of the spectral estimates of individuals - in this case animals of the same strain-in order to improve their statistical reliability.

The chi-square periodogram (Sokolove and Bushell, 1978) was used to determine the exact period length $(\tau)$ of the circadian components under different light conditions. Data were combined into 5-min bins for analysis and examined at 5 -min intervals without any interpolation procedure.

\section{Lighting Regimes of Animal Groups}

Group 1 (LD 12:12)

LA was measured continuously for 10 days under a LD cycle of $12: 12 \mathrm{~h}$ in six male rats of the following inbred strains: ACI/Ztm, AS/ $\mathrm{Ztm}, \mathrm{BH} / \mathrm{Ztm}, \mathrm{BS} / \mathrm{Ztm}$, and LEW/Ztm. The animals had been synchronized to the light-dark cycle since birth (light period from 6 AM to $6 \mathrm{PM}$ local time, light intensity 300 lux). Their age during testing was 17-20 weeks. Mean body weight at the start of measurements was as follows: 
$\mathrm{ACI} / \mathrm{Ztm}, 230 \mathrm{~g} ; \mathrm{AS} / \mathrm{Ztm}, 273 \mathrm{~g}$; BH/Ztm, $323 \mathrm{~g}$; BS/Ztm, $330 \mathrm{~g}$; and LEW/Ztm, $320 \mathrm{~g}$. Six animals could be measured simultaneously and measurements were run at random with two animals of three strains during each registration period of 10 days.

\section{Group $2(D D)$}

Locomotor activity was studied continuously for 10 days under continuous dark conditions in six male rats from each of three strains $\mathrm{ACI} /$ $\mathrm{Ztm}(200 \mathrm{~g}), \mathrm{BH} / \mathrm{Ztm}(283 \mathrm{~g})$, and LEW/Ztm (299 g), all aged $15-17$ weeks. These animals had been kept in the animal room under DD (illumination with a $60-\mathrm{W}$-red-light-lamp) since the third week after birth.

\section{Group $3(L L)$}

This group was measured continuously for 10 days under continuouslight conditions and consisted of six male rats of the strains BS/Ztm (298

g) and LEW/Ztm (340 g) and three male rats of the strains AS/Ztm (268 g) and BH/Ztm (279 g), all aged 15-17 weeks. These animals were kept 3-4 weeks under constant light before the experiments.

\section{RESULTS}

Daily Pattern of Locomotor Activity Under Light-Dark Conditions (12 $\mathrm{h}: 12 \mathrm{~h})$

The daily patterns of LA under LD (12 h: $12 \mathrm{~h})$, for six male animals of each strain, are summarized in Fig. 1. Thirty-minute mean values were computed by averaging the 5 -min values within each 30 -min interval for each day and each animal, and then by calculating a mean value of the coincident 30-min intervals of the 10-day study period for the six animals per strain. The small standard deviations between animals of the same strain, plotted as vertical lines, indicate the strong regularity of the daily LA patterns.

All five inbred strains are nocturnal. They show an activity peak at the beginning of the dark period and a, more or less, pronounced peak before light-on. LA decreases rapidly at the beginning of the light period, reaching the lowest values during the light period. It increases again toward the beginning of the dark period. During the dark period, there are obvious strain-dependent differences in the patterns of locomotor activity.

$L E W$ Three reproducible peaks occur during the dark period. The 30 -min values of LA differ from 75 to $175 \%$ of the mean activity. 

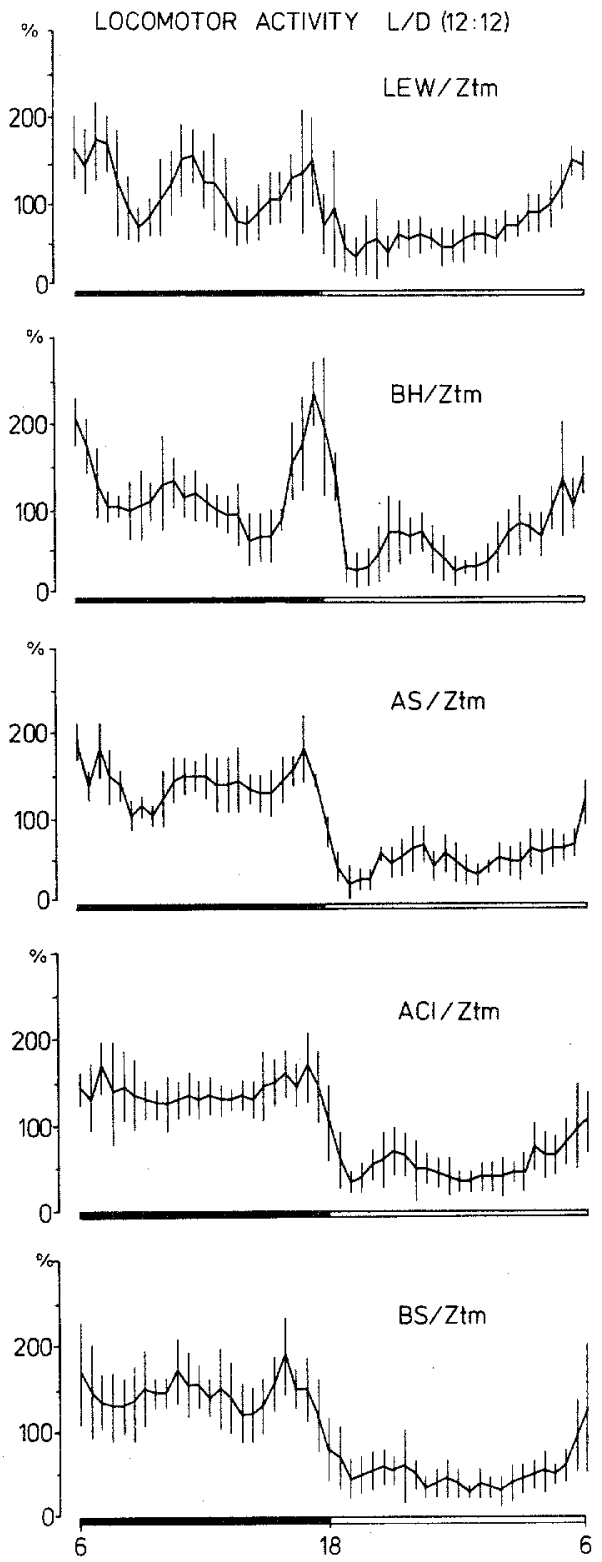

Fig. 1. Daily locomotor activity patterns of five inbred strains under LD (12 h: 12h). Thirtyminute averages taken from continuous measurements of six animals per strain over 10 days are employed as units on the graph. Standard deviations between animals are plotted as vertical lines. The black portion of the horizontal bar indicates the dark period. 

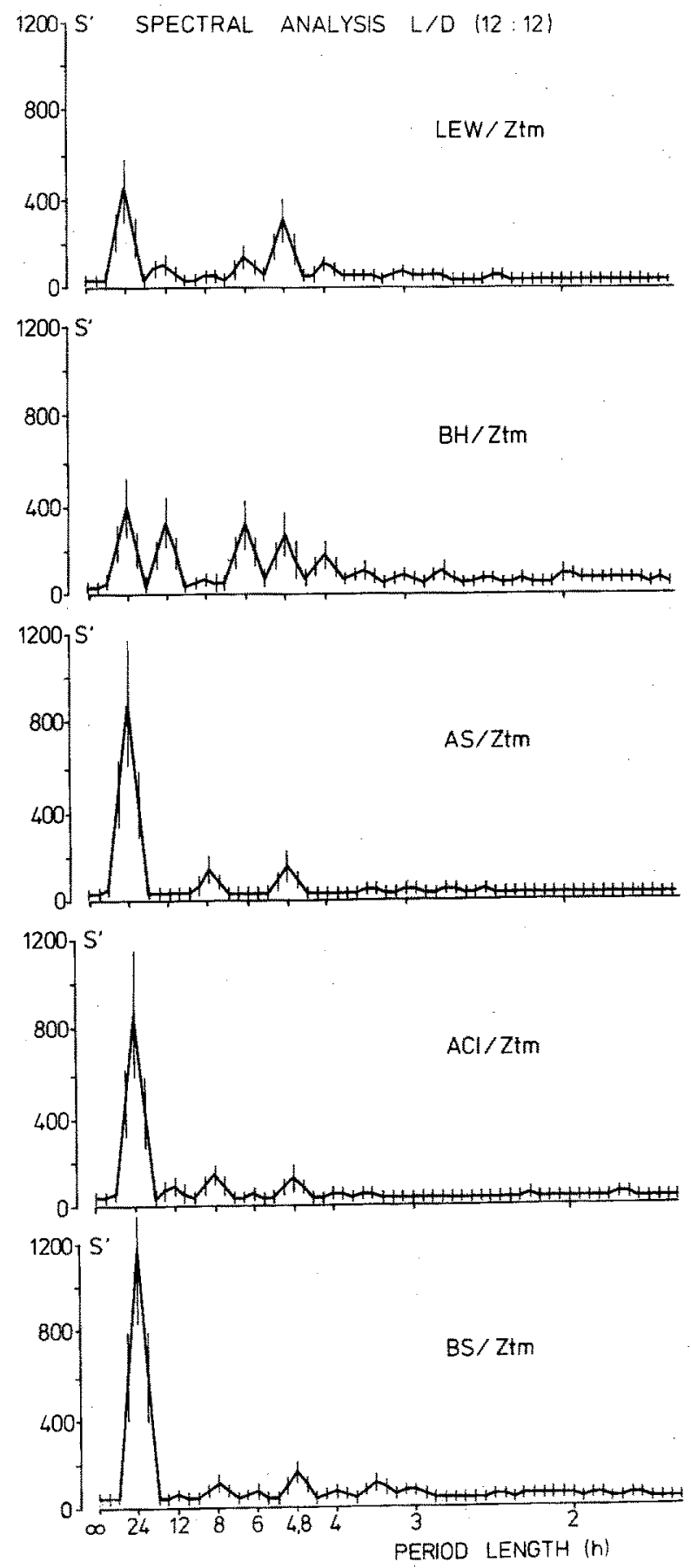
$B H$. The BH strain shows a larger variability within the dark period (65-235\% of mean activity). There are two major activity peaks, one at the beginning and one at the end of the dark period, and the two minor peaks, one at midnight and one about $4 \mathrm{~h}$ after light-on.

$A S$. The decrease in LA begins $1 \mathrm{~h}$ before light-on. Apart from this rapid decrease, the variability of LA (105-190\% of mean activity) is much smaller than in the $\mathrm{BH}$ or LEW strain.

$A C I$. A nearly constant level of LA is typical for the ACI strain. Despite the rapid decrease in LA at the end of the dark period, the ACI strain shows, compared to other strains, the smallest variations in the 30 min LA values during the night (125-170\% of mean activity).

$B S$. The BS strain shows an early decrease in LA $2 \mathrm{~h}$ before lighton. The variability of LA (120-190\% of mean activity) during the dark period is nearly the same as in the AS strain.

These differences, obvious in the daily patterns of the five strains, as shown in Fig. 1, were examined by a one-way analysis of variance and according to Student's $t$-test. This was done by comparing the coincident 30-min mean values of each of the animals' daily profiles.

The results of the 10 strain comparisons are arranged in Table I. The first four comparisons are made against LEW, the next three against $\mathrm{BH}$, and the final three among AS, ACI, and BS. Significant differences between the LEW and the AS, ACI, and BS strains are obvious at 21.00$22.00,01.30-04.00$, and 12.00-18.00 h. Comparisons with the BH strain indicate significant differences regarding the LA of the ACI, AS, and BS strains and these are concentrated between 23.00 and $6.30 \mathrm{~h}$. The last three comparisons among the ACI, AS, and BS strains indicate relatively minimal differences.

\section{Spectral Analysis of Locomotor Activity Under Light-Dark Conditions}

$(12 \mathrm{~h}: 12 \mathrm{~h})$

Fig. 2 shows the spectral analysis of LA of the five strains under LD $(12 \mathrm{~h}: 12 \mathrm{~h})$. Each spectrum is a result of data pooled from six animals per strain. The $95 \%$ confidence limits of the spectral estimates are plotted as vertical lines. The AS, ACI, and BS strains show a clear 24-h component. This component is two times, in the case of $\mathrm{AS}$ and $\mathrm{ACI}$, or three times, in the case of BS, greater than the 24-h component of the LEW and $\mathrm{BH}$ strains. The spectral analysis of the LEW and $\mathrm{BH}$ strains includes

Fig. 2. Spectral analysis of locomotor activity within five inbred strains under LD (12:12). The $95 \%$ confidence limits of the spectral estimates of six animals per strain are plotted as vertical lines. 


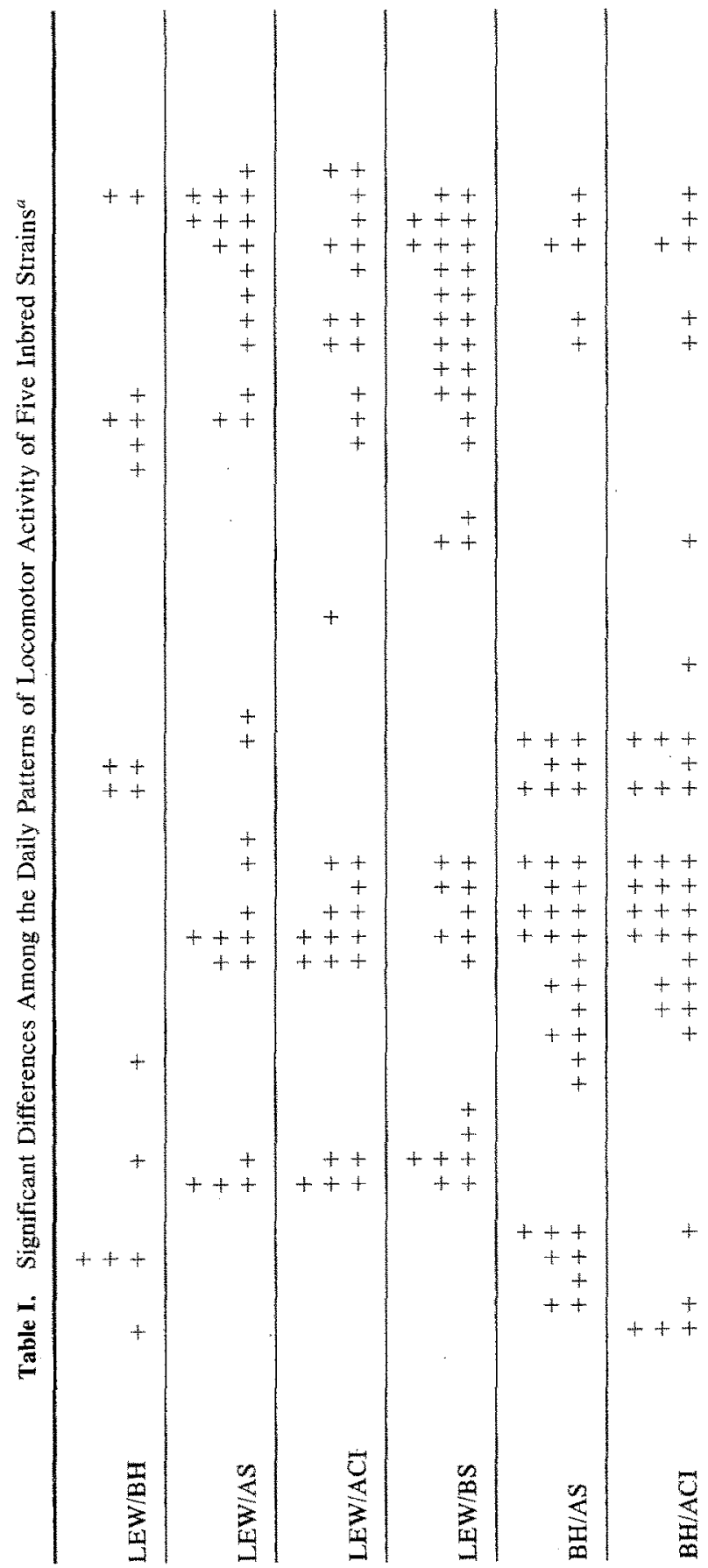




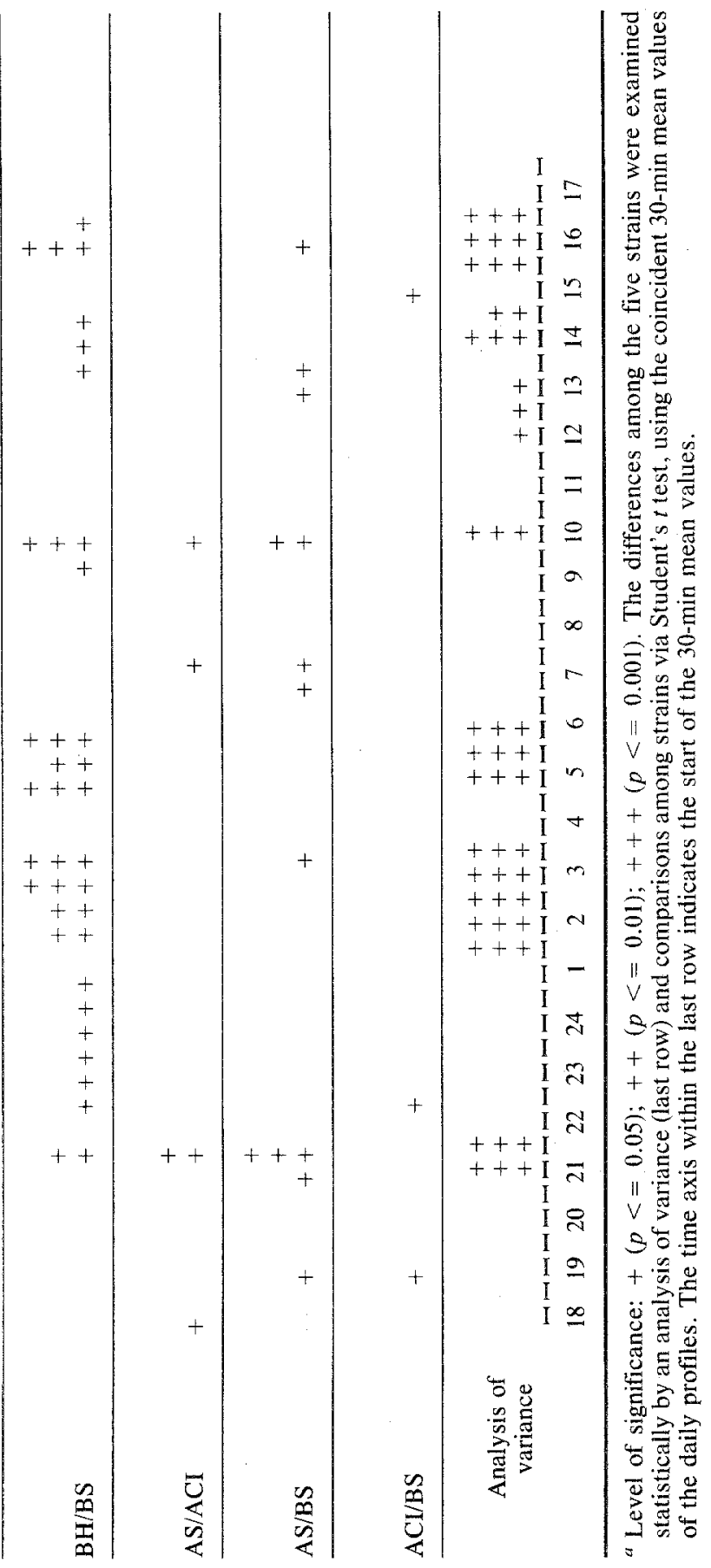


several components of less than $24 \mathrm{~h}$. The LEW strain shows a clear component of 4.8-h period length. This was already evident from the three activity peaks during the night (Fig. 1). The BH strain exhibits a 12-h rhythm in the variance spectra, corresponding to the bimodal pattern of LA, as seen in Fig. 1. Additional rhythms of $6,4.8$, and $4 \mathrm{~h}$, although less evident in the daily pattern, can be noted. The ACI, AS, and BS strains also show rhythms of 8 and $4.8 \mathrm{~h}$. The contribution of these rhythms to the variability of LA, however, is relatively small compared with the contribution of the circadian components.

\section{Locomotor Activity Under Continuous-Dark (DD) Conditions}

Fig. 3 shows the variance spectra of LA under DD for the strains LEW, BH, and ACI. A comparison between the variance spectra obtained from measurements under $\mathrm{LD}$ and $\mathrm{DD}$ reveals no considerable modification of the typical strain pattern. The 24-h rhythm persists, as well as the other components found in the LEW and the BH strain under LD.

In the LEW strain, no considerable change in the relative contribution of the 24- and 4.8-h rhythm is observed. There is an increase, however, in the amplitudes of the 12-, the 8-, and above all, the 4-h, rhythms. The variance spectra for the $\mathrm{BH}$ strain are also nearly identical under LD and DD. Only the $24-$ and 12 -h rhythms are slightly reduced. In the ACI strain, the 24-h rhythm is also reduced under DD, while the rhythms of $12,8,6$, and $4.8 \mathrm{~h}$ are increased by only a small amount.

\section{Locomotor Activity Under Continuous-Light (LL) Conditions}

Under LL, considerable modifications of the typical LA patterns of each strain are evident, although these measurements took place after a short adaptation to continuous light of only 3 to 4 weeks. Figure 4 demonstrates the variance spectra under LL for the strains LEW(six animals), BH(three animals), AS(three animals), and BS(six animals). With the exception of the BH strain, all other strains show a marked decrease in the circadian rhythm, while a broad spectrum of ultradian rhythms persists in the LEW and BH strains. This is particularly noticable in Fig. 5, showing the typical activity pattern of a single rat (LEW) under LL. Bursts of activity are distributed over the whole day. Spectral analysis of this data reveals no circadian rhythm, rather only significant ultradian rhythms in the range of 4 to $6 \mathrm{~h}$, with a mean peak at $5.3 \mathrm{~h}$. The same is true for two of the three animals of the $\mathrm{BH}$ strain measured under LL. They likewise showed only clear ultradian peaks and no significant circadian rhythms. 

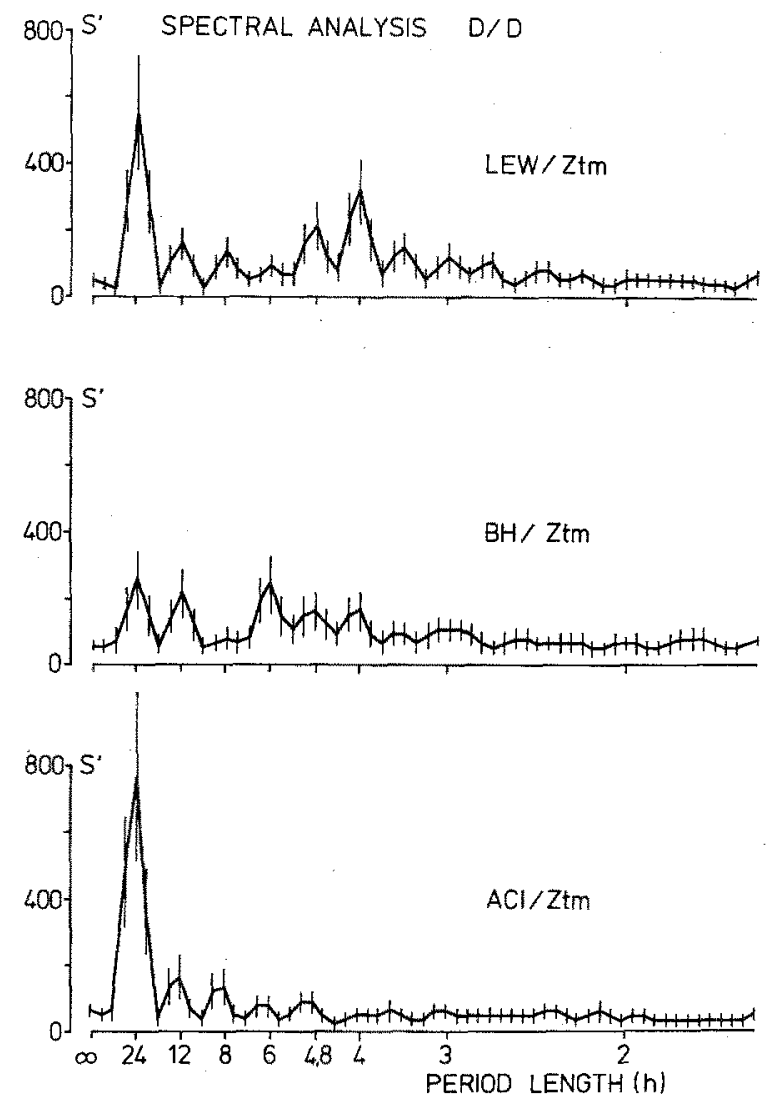

Fig. 3. Spectral analysis of locomotor activity within three inbred strains under DD. The $95 \%$ confidence limits of the spectral estimates of six animals are plotted as vertical lines.

These details are lost in the pooled variance spectra shown in Fig. 4. As a result of the great variability. within and between the animals, they reveal only a broad spectrum of rhythms in the range of 3 to $6 \mathrm{~h}$ in the LEW strain and 2 to $8 \mathrm{~h}$ in the BH strain. The AS and BS strains failed to show any rhythmic patterns under LL. Neither significant circadian nor significant ultradian rhythms can be shown within the variance spectra.

\section{Period Length of the Circadian Rhythms Under Light-Dark (LD) and Continuous-Dark (DD) Conditions}

The calculated period lengths $(\tau)$ for each light condition are summarized in Table II. Under LD, all strains show a nearly perfect $24-\mathrm{h}$ 

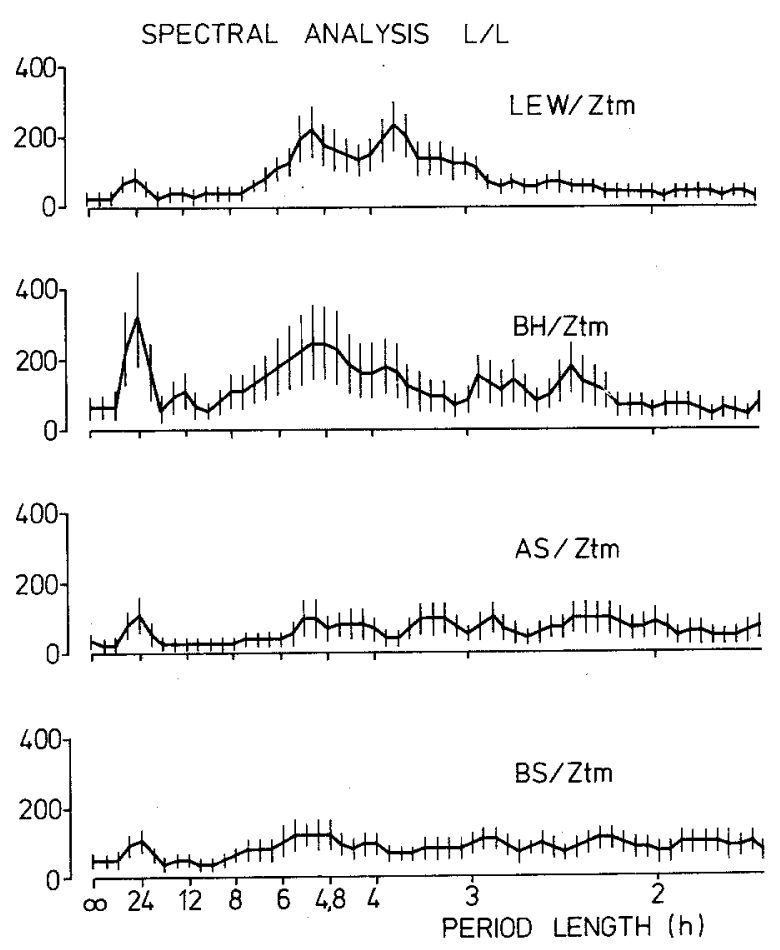

Fig. 4. Spectral analysis of locomotor activity under LL for the strains LEW(six animals), BH(three animals), AS(three animals), and BS(six animals). The 95\% confidence limits of the spectral estimates are plotted as vertical lines.

rhythm. Under DD, the LEW strain shows only a slightly significant ( $p$ $<=0.05)$ lengthening of the 24-h component $(\tau D D=24 \mathrm{~h} 7 \mathrm{~min})$. The $\mathrm{BH}$ and $\mathrm{ACI}$ strains exhibit free-running circadian rhythms of $\tau \mathrm{DD}=25$ $\mathrm{h} 16 \mathrm{~min}(\mathrm{BH})$ and $\tau \mathrm{DD}=24 \mathrm{~h} 44 \mathrm{~min}(\mathrm{ACI})$. These differences are highly significant ( $p<=0.001$ ) compared to $\tau$ LD. Under LL, all strains show a rapid decrease in the amplitude of the circadian rhythm. The estimates of $\tau \mathrm{LL}$ are not shown in Table II. This is because only two animals of the LEW strain, and one animal of the BH strain, exhibit significant circadian rhythms when analyzed according to the chi-square test. Nevertheless, the significant, as well as, the nonsignificant circadian components tend to lengthen under LL.

The two LEW rats exhibit a free-running period of $\tau \mathrm{LL}=26 \mathrm{~h} 15$ min and $\tau \mathrm{LL}=25 \mathrm{~h} 0 \mathrm{~min}$. The animal of the $\mathrm{BH}$ strain exhibits $\tau \mathrm{LL}=$ $25 \mathrm{~h} 20 \mathrm{~min}$. 


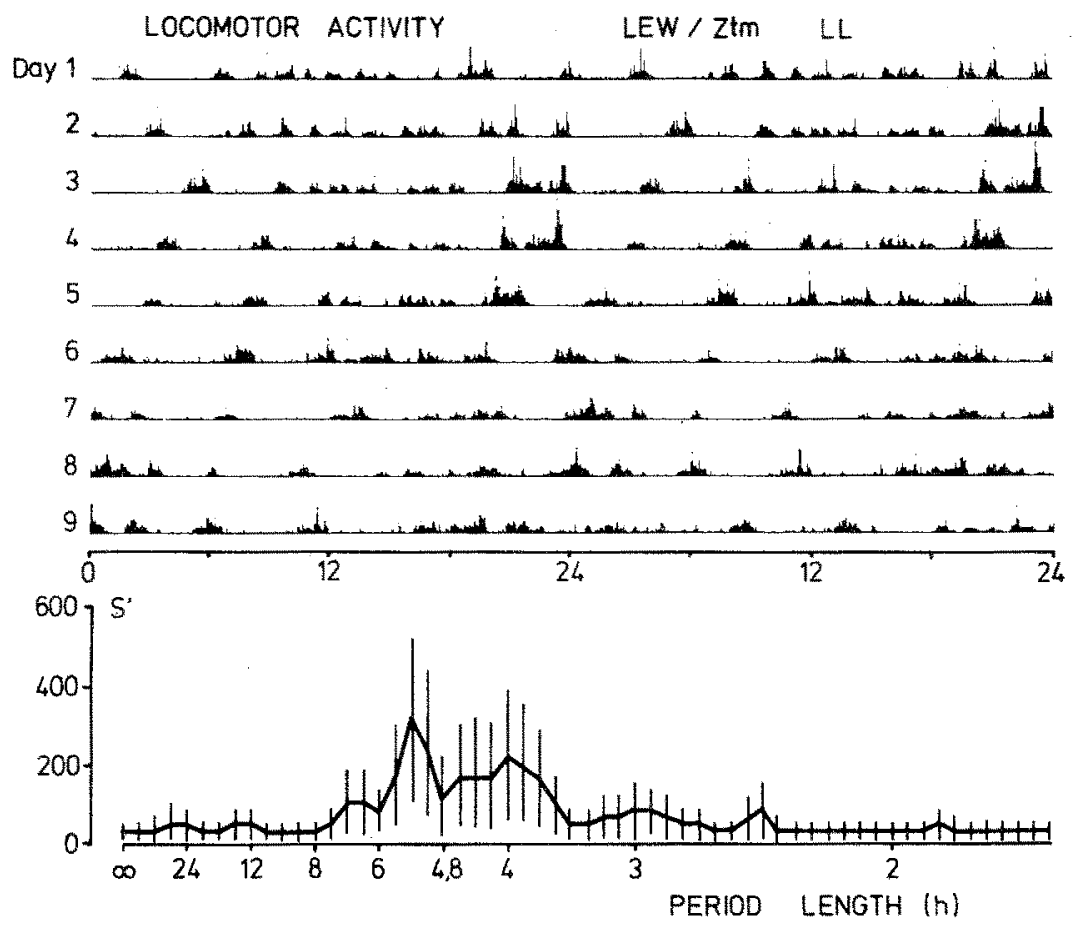

Fig. 5. Top: Locomotor activity pattern of a single rat (LEW) under LL, double plotted over 10 days. Bottom: Spectral analysis of the same animal.

\section{DISCUSSION}

Inbred strain comparisons provide a convenient method for separating components of genetic and environmental variance. Individuals from the same strain are genetically identical, and, therefore, differences within strains reflect environmental effects and/or errors in measurement.

This study showed pronounced strain differences in the laboratory rat with regard to the daily pattern of LA under a light-dark regime of 12:12 hours (Fig. 1). Since the variability of the daily patterns is very small within strains, these differences can be considered predominately genetic in nature. Similar daily patterns of LA were found by Lemmer et al. (1981) for the ACI, BS, and LEW strains. The reported pattern of the $\mathrm{BH}$ strain, however, differs from our results. It should be noted that the measurements of Lemmer et al. were conducted on all strains for 2 to 4 days only and with groups of five rats per cage. Despite these differences in the measuring technique, their data, likewise, indicate genetically fixed differences. 
Table II. Mean Values ( $\tau$ ) and Standard Deviations (in Parentheses) of the Period Length of the 24-h Rhythms Under Light-Dark (LD) and Continuous-Dark (DD) Conditions

\begin{tabular}{|c|c|c|}
\hline & $\tau L D$ & TDD \\
\hline \multirow[t]{2}{*}{ LEW } & $\begin{array}{c}24 \text { h } 00 \min ( \pm 0 \mathrm{~min}) \\
n=6\end{array}$ & $\begin{array}{c}24 \text { b } 07 \min ( \pm 8 \mathrm{~min}) \\
(\mathrm{n})=6\end{array}$ \\
\hline & \multicolumn{2}{|c|}{$p<=0.05$} \\
\hline \multirow[t]{2}{*}{ BH } & $\begin{array}{c}24 \mathrm{~h} 00 \min ( \pm 0 \mathrm{~min}) \\
n=6\end{array}$ & $\begin{array}{c}24 \text { h } 16 \min ( \pm 6 \min ) \\
n=6\end{array}$ \\
\hline & \multicolumn{2}{|c|}{$p<=0.001$} \\
\hline \multirow[t]{2}{*}{$\mathrm{ACl}$} & $\begin{array}{c}23 \text { h } 59 \min ( \pm 9 \min ) \\
n=6\end{array}$ & $\begin{array}{c}24 \text { h } 44 \min _{n=6}( \pm 6 \min ) \\
\text { (n) }\end{array}$ \\
\hline & \multicolumn{2}{|c|}{$p<=0.001$} \\
\hline AS & $\begin{array}{c}24 \text { h } 01 \min _{n=6}( \pm 2 \min ) \\
n=6\end{array}$ & \\
\hline BS & $\begin{array}{c}23 \mathrm{~h} 55 \min ( \pm 6 \min ) \\
n=6\end{array}$ & \\
\hline
\end{tabular}

Further evidence supporting genetically based differences in LA rhythms of rats can be found in a recent study done by Stephan (1983). He described the activity pattern in an outbred Sprague-Dawley stock under DD as nearly unimodal or weakly bimodal. In addition, he observed trimodal, as well as bimodal, activity distributions in a small number of animals. The strain differences found in the LA patterns under LD are obvious in the spectral analysis (Fig. 2) and persisted under continuousdark conditions (Fig. 3). Since the only convincing evidence of an endogenous periodicity is the free-running rhythm (Aschoff, 1960), the actual length of the circadian period under different light conditions was estimated (Table II). Under LD, all strains show a nearly perfect $24-\mathrm{h}$ rhythm. The mean period length deviates approximately 1 min in the ACI and AS strains and about $5 \mathrm{~min}$ in the BS strain. This may be due to data sampling and/or the compilation of the data into 5-min intervals. It may also have biological explanation such as the drifting of the mean activity peaks during the testing period. Under continuous dark, there are clear free-running rhythms for the BH ( TDD $=24 \mathrm{~h} 16 \mathrm{~min}$ ) and the ACI ( $\mathrm{TDD}$ $=24 \mathrm{~h} 44 \mathrm{~min}$ ) strains, while the LEW strain shows only a small lengthening of its period $(\tau \mathrm{DD}=24 \mathrm{~h} 7 \mathrm{~min})$.

These results correspond well to those reported for Sparque-Dawley rats $[\tau D D=24$ h $10 \mathrm{~min}$ (Gibbs, 1979); 24 h $18 \mathrm{~min}$ (Szafarczyk et al., 1981); 24 h 5 min (Summer and McCormack, 1982) ] and pigmented rats of LEWIS/BN hybrids [TDD $=24 \mathrm{~h} 6 \mathrm{~min}$ (Terman and Terman, 1980)].

It should be mentioned that long-term observations over several months indicate considerable changes in DD. In addition to small long- 
term changes, $\tau \mathrm{DD}$ is influenced by prior entrainment to LD cycles and ambient temperature (Aschoff, 1979). Despite these limitations, several studies report a "preferred" $\tau$ DD value $>24$ h for the rat.

Because of the arhythmic character of LA under LL, these measurements must be considered separately. Free-running circadian rhythms can be observed in many organisms only in continuous darkness or constant dim illumination. Increasing light intensities lengthen the rhythms in relation to DD (Aschoff, 1979) and also alter some other parameters (Aschoff and Wever, 1962). Under continuous bright light with intensities corresponding to that of normal daylight, most organisms are arhythmic (Wever, 1980).

The LA patterns of the AS and BS strains tend to be arhythmic under LL. Spectral analysis shows neither significant ultradian nor significant circadian rhythms. With the exception of three animals, the LEW and BH strains showed a markedly decreased circadian, but unchanged ultradian rhythms under LL.

The analysis of ultradian rhythms in the presence of a circadian rhythm is a difficult problem. Ultradian components observed in this study are to some extent the result of the harmonic decomposition of a 24-h square wave. This could be the case with such periods in the ACI, AS, and BS strains but does not, however, explain the appearance of such ultradian components in the LEW and $\mathrm{BH}$ strains.

In the LEW strain, the 4.8-h period corresponds well with the three peaks of activity during the night phase. The 12-h rhythm of the BH strain is in agreement with the bimodal activity pattern. Such noncircadian periods can be interpreted as two (BH) or three (LEW) 24-h oscillations which are $12(\mathrm{BH})$ or $4.8(\mathrm{LEW}) \mathrm{h}$ out of phase. The continuance of the ultradian rhythms under LL in the LEW and BH strains, as shown for a single LEW rat in Fig. 5, supports the hypothesis that there is an independent ultradian oscillator causing the activity rhythms in the LEW and BH strains.

The results shown in this study do not totally elucidate this problem. Further investigations involving inbred strains as models of various biorhythmical behavior will be needed to understand the concept of the biological clock in the rat.

\section{REFERENCES}

Aschoff, J. (1960). Exogenous and endogenous components in circadian rhythms. Cold Spring Harbor Symp. Quant. Biol. 25:11-28.

Aschoff, J. (1979). Circadian rhythms: Influences of internal and external factors on the period measured in constant conditions. Z. Tierpsychol. 49:225-249.

Aschoff, J, and Wever, R. (1962). Aktivitätsmenge und $\alpha: \rho$ Verhältnis als Meßgröße der Tagesperiodik. Z. vergl. Physiol. 46:88-101. 
Büttner, D., and Plonait, H. (1980). Langfristige Messungen der maximalen, mittleren und Ruheherzfrequenz an Laborratten mittels implantierbarer Telemetriesender. Zentral. Veterinärmed. A27:269-278.

Büttner, D., and Wollnik, F. (1982). Spontaneous short-term fluctuations in the daily pattern of heart rate, body temperature and locomotor activity in the laboratory rat. Lab. Anim. 16:319-326.

Daan, S. (1981). Adaptive daily strategies in behavior. In Aschoff, J. (ed.), Handbook of Behavioral Neurobiology, Vol. 4, Biological Rhythms, pp. 275-298.

Gibbs, F. P. (1979). Fixed interval feeding does not entrain the circadian pacemaker in blind rats. Am.J. Physiol. 236:R249-R253.

GV-Solas (1977). Liste von Erregern zur Spezifizierung bei SPF-Versuchstieren. Veröffentlichungen der Gesellschaft für Versuchstierkunde Nr. 2.

Feldman, J. F., and Hoyle, M. N. (1973). Isolation of circadian clock mutants of neurospora crassa. Genetics 75:605-613.

Halberg, F., and Panofsky, H. (1961). I. Thermo-variance spectra; Method and clinical illustrations. Exp. Med. Surg. 19:285-309.

Honma, K., and Hiroschige, T. (1978a). Simultaneous determination of circadian rhythms of locomotor activity and body temperature in the rat. Jap. J. Physiol. 28:159-169.

Honma, K., and Hiroshige, T. (1978b). Internal synchronization among several circadian rhythms in rats under constant light. Am. J. Physiol. 235(5):R243-R249.

Honma, K., and Hiroshige, T. (1978c). Endogenous ultradian rhythms in rats exposed to prolonged continuous light. Am. J. Physiol. 235(5):R250-R256.

Konopka, R. J., and Benzer, S. (1971). Clock mutants of drosophila melanogaster. Proc. Natl. Acad. Sci. USA 68:2112-2116.

Lemmer, B. G., Caspari-Irving, G., and Weimer, R. (1981). Strain-dependency in motor activity and in concentration and turnover of catecholamines in synchronized rats. Pharmacol. Biochem. Behav. 15:173-178.

Panofsky, H., and Halberg, F. (1961). II. Thermo-variance spectra; Simplified computational example and other methodology. Exp. Med. Surg. 19:323-338.

Peleg, L., Nesbitt, M. N., and Ashkenazi, I. E. (1982). A strain difference in the daily rhythm of glyceraldehyde-3-phosphate dehydrogenase activity in the mouse. J. Comp. Physiol. 148:137-142.

Plonait, H., Büttner, D., and Müschen, U. (1982). Ein kapazitiv arbeitendes System zur Messung der Bewegungsaktivität kleiner Labortiere. Z. Versuchstierkunde 24:244-249.

Possidente, B., and Hegman, J. P. (1980). Circadian rhythms under common gene control. J. Comp. Physiol. 139:121-125.

Sokolove, P. G., and Bushell, W. N. (1978). The chi-square periodogram: Its utility for analysis of circadian rhythms. J. Theor. Biol. 72:131-160.

Stephan, F. K. (1983). Circadian rhythms in the rat: constant darkness, entrainment to T cycles and to skeleton photoperiods. Physiol. Behav. 30:451-462.

Summer, T. L., and McCormack, C. E. (1982). Phase-response-curve (PRC) of circadian locomotor activity of the rat. Fed. Proc. 41:1698.

Szafarcyk, A., Ixart, G., Alonso, G., Malaval, F., Nouguier-Soule, J., and Assenmacher, 1. (1981). Neural control of circadian rhythms in plasma ACTH, plasma corticosterone and motor activity. J. Physiol. (Paris) 77:969-976.

Terman, J. S., and Terman, M. (1980). Effects of illumination level on the rat's rhythmicity of brain self-stimulation behavior. Behav. Brain. Res. 1:507-519.

Wever, R. A. (1980). Circadian rhythms of finches under bright light: Is self-sustainment a precondition for circadian rhythmicity? J. Comp. Pysiol. 139:49-58. 